(Supporting Information)

\title{
Graphene-Based Dual-Metal Sites for Oxygen Reduction Reaction: A Theoretical Study
}

\author{
Jiayi $\mathrm{Xu}^{1 *}$, Ayyappan Elangovan² ${ }^{2}{\mathrm{Jun} \mathrm{Li}^{2}, \text { Bin Liu }}^{1 *}$
}

${ }^{1}$ Tim Taylor Department of Chemical Engineering, Kansas State University, Manhattan, KS, 66506, USA

${ }^{2}$ Department of Chemistry, Kansas State University, Manhattan, KS, 66506, USA

*Corresponding author: jasonxjy@ksu.edu, binliu@ksu.edu 


\section{Table of Contents}

Figure S1. Optimized dual-metal sites (consisting of PGM) and configurations of $\mathrm{O}_{2}, \mathrm{OOH}, \mathrm{O}$, and $\mathrm{OH}$ adsorbates. Color code: brown-C, light blue-N, yellow-Fe, dark blue-Co, pink-Ni, purple$\mathrm{Cu}$, silver-Pt, green-Pd, red-O, and white, $\mathrm{H}$.

Figure S2. Optimized dual-metal sites (consisting of non-PGM only) and configurations of $\mathrm{O}_{2}$, $\mathrm{OOH}, \mathrm{O}$, and $\mathrm{OH}$. Color code: brown-C, light blue-N, yellow-Fe, dark blue-Co, pink-Ni, purple$\mathrm{Cu}$, red-O, and white, $\mathrm{H}$.

Figure S3. Optimized dual-metal site and configurations $\mathrm{O}_{2}, \mathrm{OOH}, \mathrm{O}$, and $\mathrm{OH}$ that bind on the opposite side of the pre-adsorbed $\mathrm{OH}$ ligand. Color code: brown-C, light blue-N, yellow-Fe, dark blue- $\mathrm{Co}$, pink-Ni, purple- $\mathrm{Cu}$, silver-Pt, red-O, and white-H.

Figure S4. Bader charge analysis of Fe-Fe (a), Fe-Co (b), Fe-Ni (c), Fe-Cu (d), Co-Co (e), Co-Ni (f), and Pt-Co (g) with (right) and without (left) the pre-adsorbed OH ligand. Color code: brown$\mathrm{C}$, light blue-N, yellow-Fe, dark blue-Co, pink-Ni, purple-Cu, silver-Pt, red-O, and white, $\mathrm{H}$.

Figure S5. Optimized dual-metal site and configurations $\mathrm{O}_{2}, \mathrm{OOH}, \mathrm{O}$, and $\mathrm{OH}$ that bind on the same side of the pre-adsorbed $\mathrm{OH}$ ligand. Color code: brown-C, light blue-N, yellow-Fe, dark blue-Co, pink-Ni, purple-Cu, silver-Pt, red-O, and white-H.

Figure S6. Free energy diagrams of ORR over (a) $\mathrm{Fe}-\mathrm{Fe}(\mathrm{OH})_{\mathrm{s}}$, (b) $\mathrm{Fe}-\mathrm{Co}(\mathrm{OH})_{\mathrm{s}}$, (c) $\mathrm{Fe}-\mathrm{Ni}(\mathrm{OH})_{\mathrm{s}}$, (d) $\mathrm{Fe}-\mathrm{Cu}(\mathrm{OH})_{\mathrm{s}}$, (e) $\mathrm{Co}-\mathrm{Co}(\mathrm{OH})_{\mathrm{s}}$, (f) $\mathrm{Co}-\mathrm{Ni}(\mathrm{OH})_{\mathrm{s}}$, and $(\mathrm{g}) \mathrm{Pt}-\mathrm{Co}(\mathrm{OH})_{\mathrm{s}}$ at the same side of $\mathrm{OH}$ ligand. "*” indicates clean surface.

Figure S8. The relationship between the adsorptions of $\mathrm{OH}$ and $\mathrm{O}_{2}$ (i.e., $\Delta G_{O H^{*}}$ and $\Delta G_{O_{2}^{*}}$ ).

Table S1. Adsorption free energies (in $\mathrm{eV}$ ) of ORR intermediates and onset potential $\left(U_{\text {lim }}\right.$ in V) on the same side of pre-adsorbed $\mathrm{OH}$. 


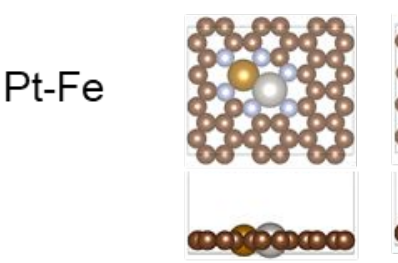

Pt-Co
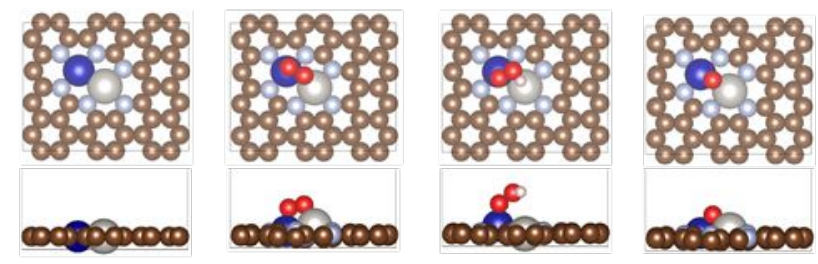

oxionenos

nగifocon
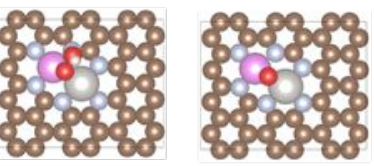

Pt-Ni
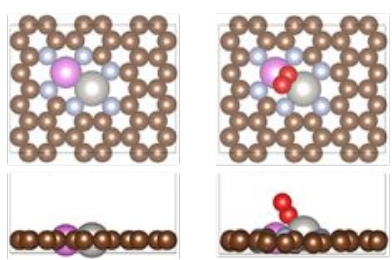

18000000

nos.

106ి0000
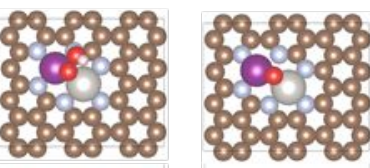

o 1660000
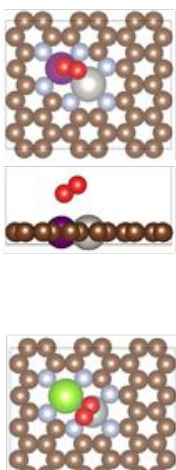

Pd-Ni

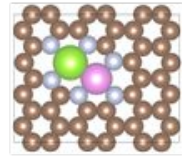

noreconem

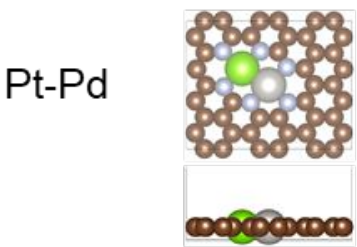

8

Figure S1
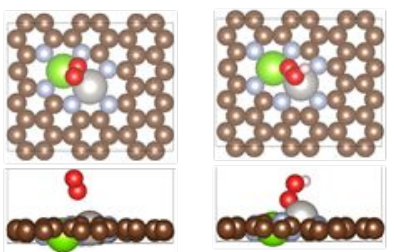

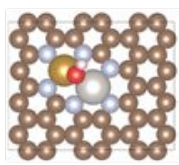

1060000

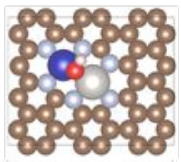

nctioneco

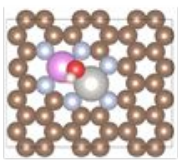

.060000

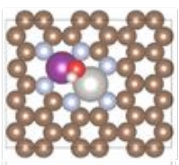

1060000
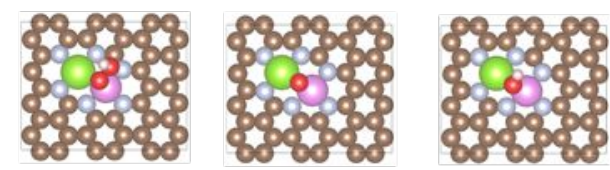

nerosenes

అంீిలు

10ㅇำ

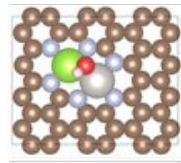

1000000 


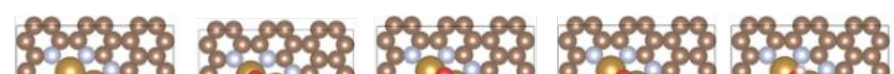

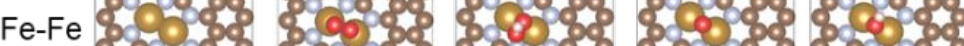

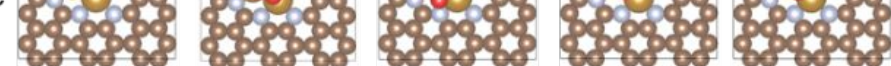
-

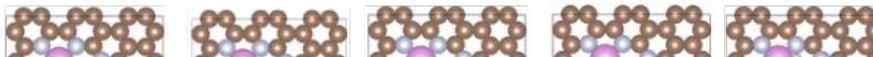
Fe-Ni $50 \%$ \% $5 \%$

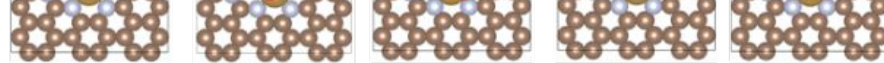

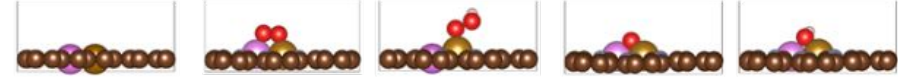

\$28క Co-Co\$05\% \$.5\% $0.5 \%$

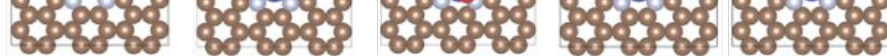

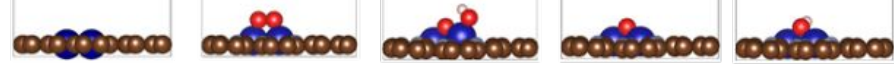

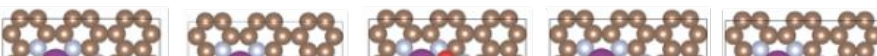
Co-Cu $\$ 05 \%$ \% $05 \%$ \$35.8

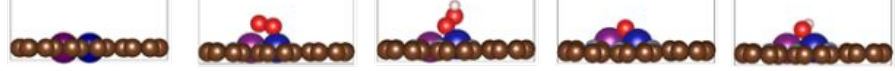

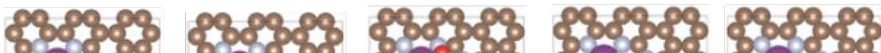
Ni-Cu $\$ 0 \%$ \% $\%$ \% 3858\% Figure S2

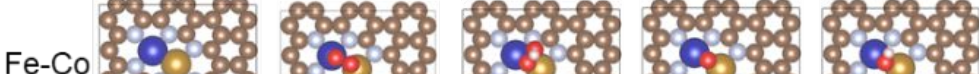

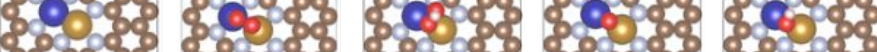

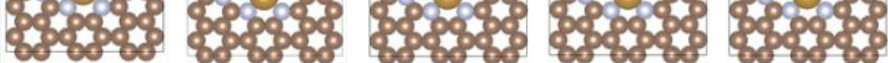

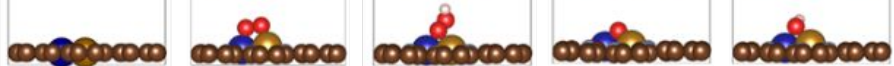

\%8.

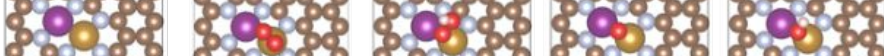
$58358 \% 585 \%$ 1,

50\%ా Ni $0.5 \%$

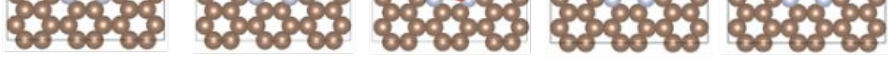
ம

Ni-Ni \%os\% 20\%

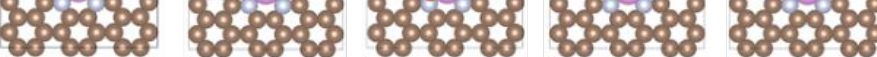

ద

\$8

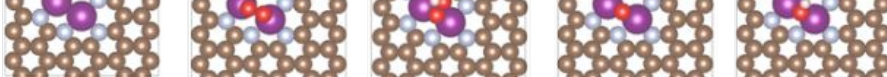

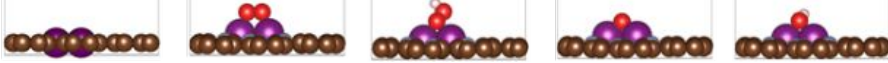
Xu et al. 


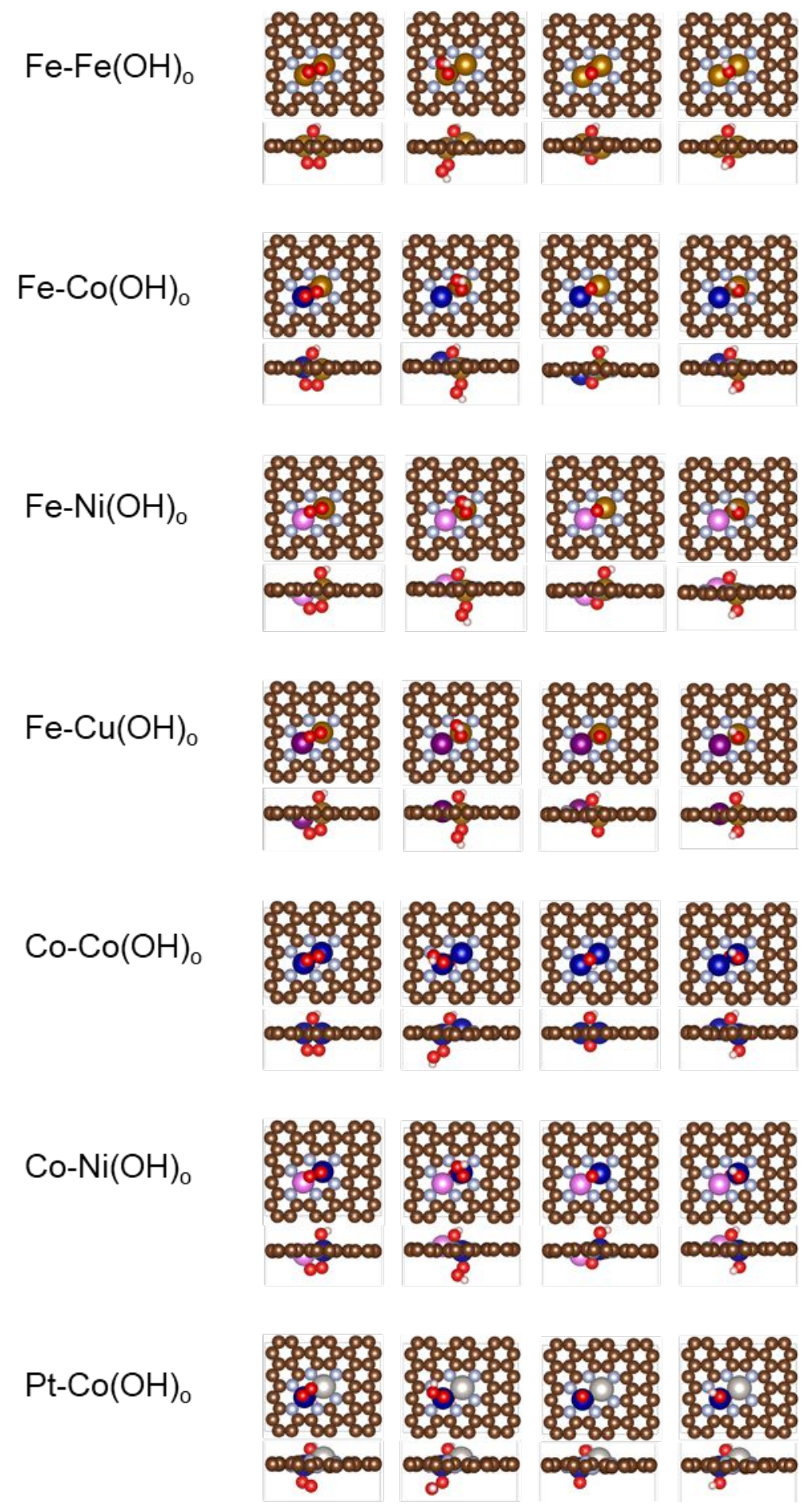

Figure S3

Xu et al. 

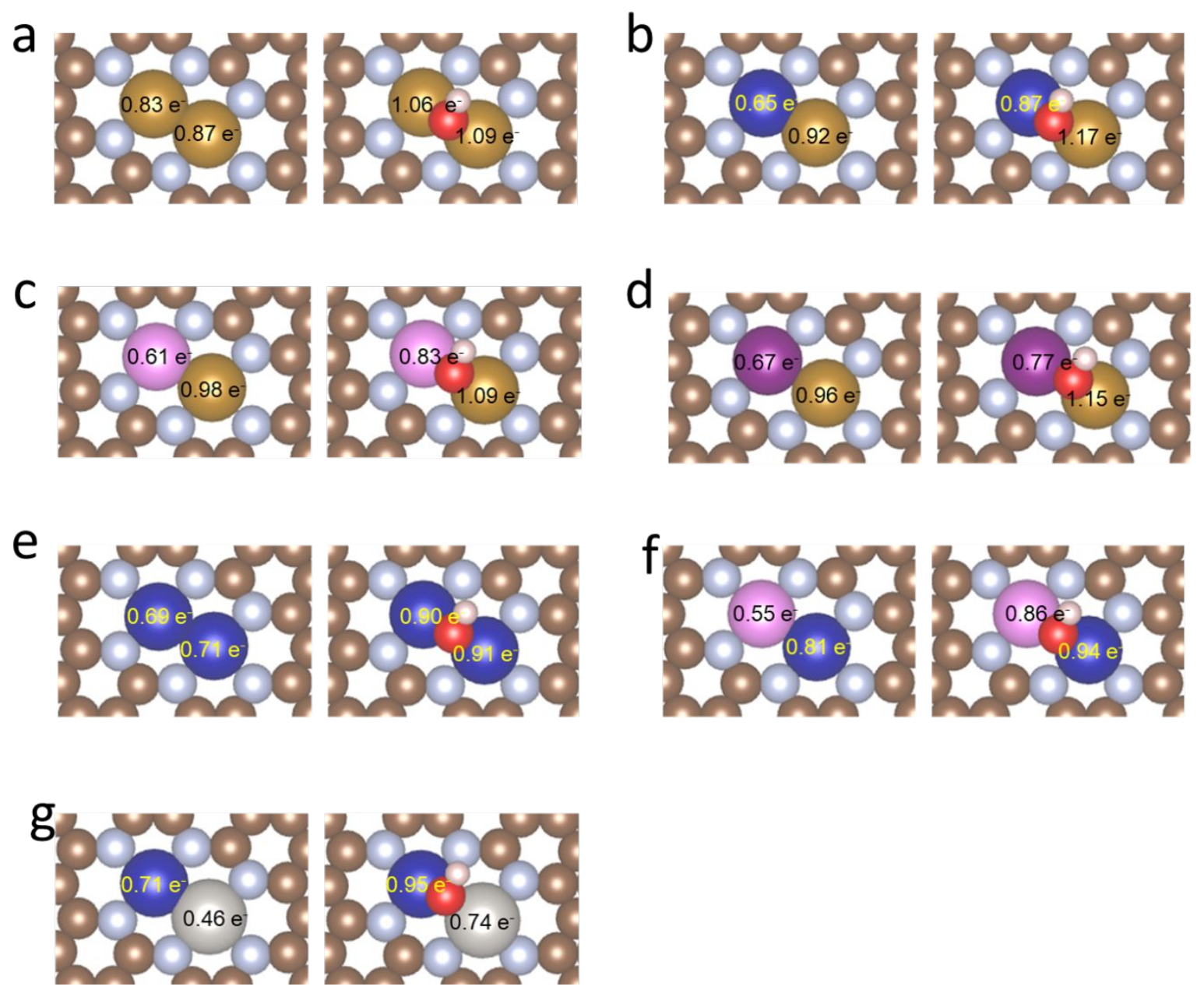

Figure S4

Xu et al. 


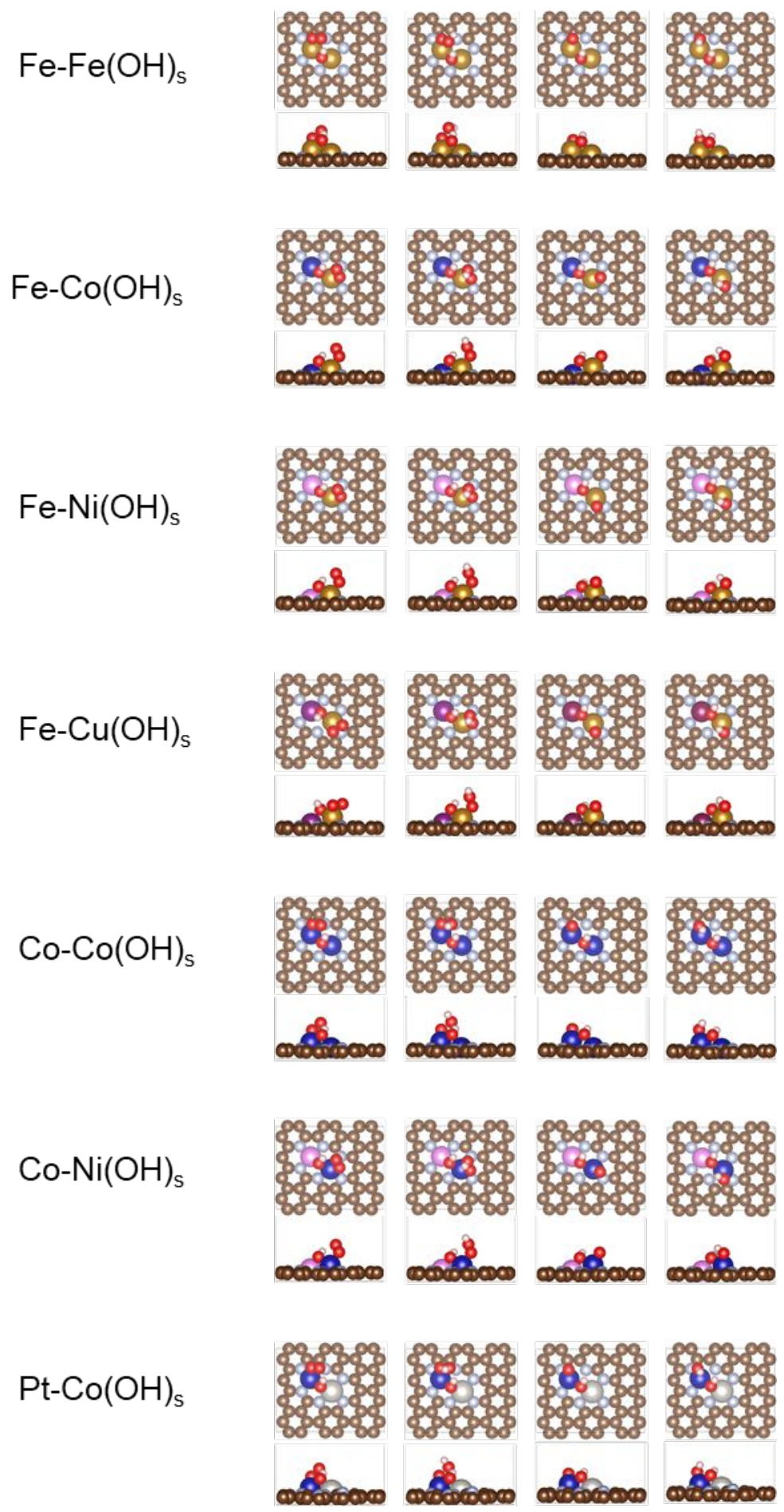

Figure S5

Xu et al. 


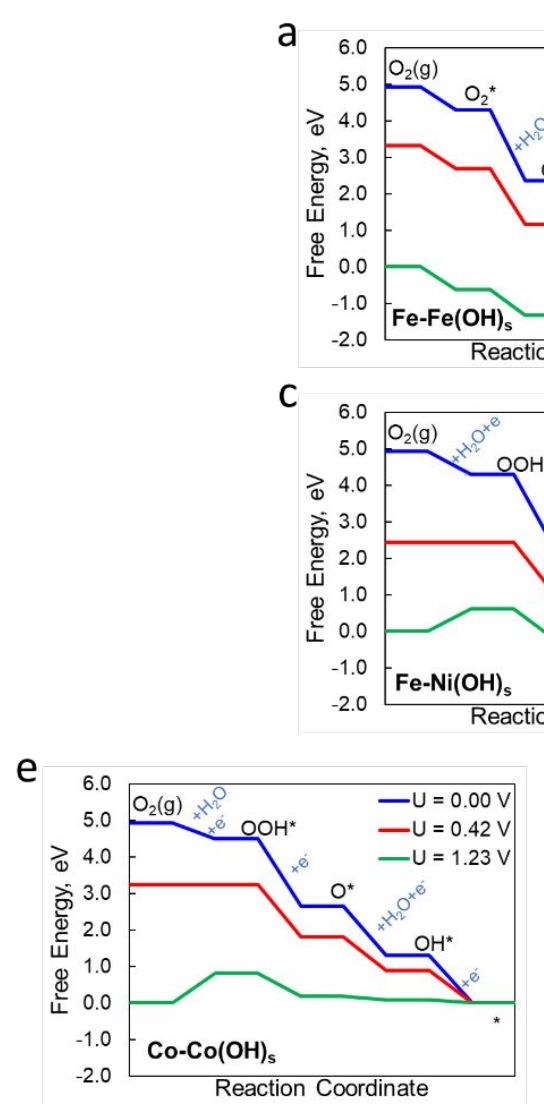

Figure S6

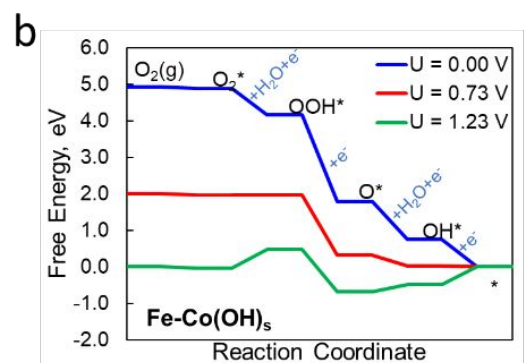

d

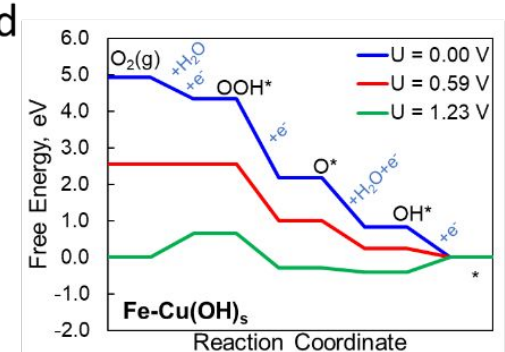

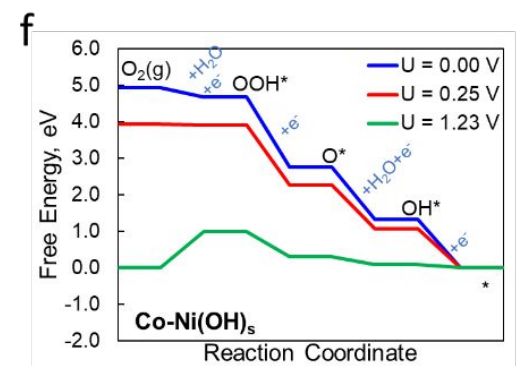

Reaction Coordinate

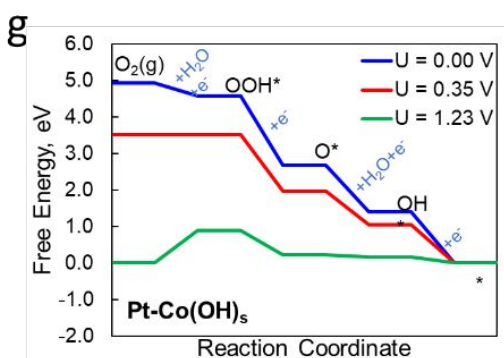

Xu et al. 


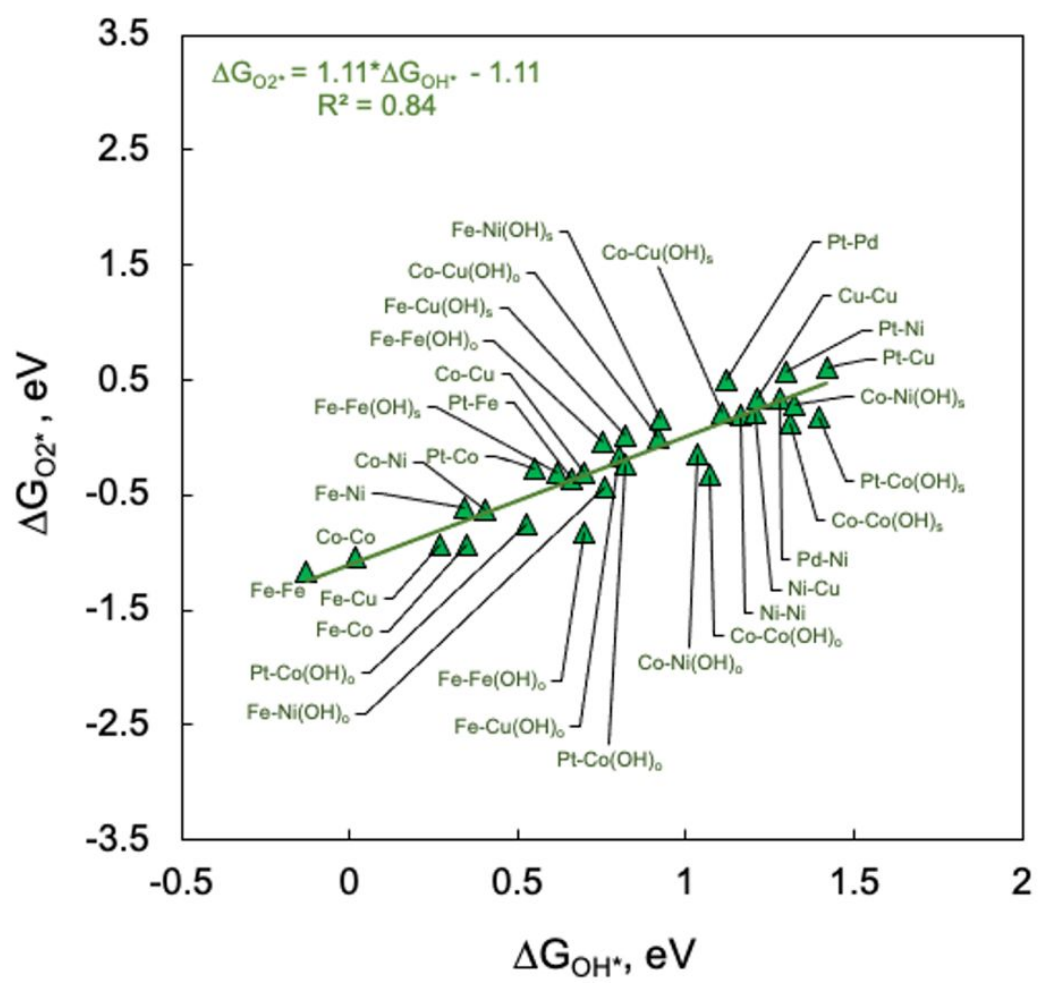

Figure S7

Xu et al. 


\begin{tabular}{ccccccc}
\hline & $\Delta G_{O_{2}^{*}}$ & $\Delta G_{O O H^{*}}$ & $\Delta G_{O^{*}}$ & $\Delta G_{O H^{*}}$ & $U_{\text {lim }}$ & RLS \\
\hline $\mathrm{Fe}-\mathrm{Fe}(\mathrm{OH})_{\mathrm{s}}$ & -0.31 & 3.90 & 1.55 & 0.62 & 0.62 & $\mathrm{R} 7$ \\
$\mathrm{Fe}-\mathrm{Co}(\mathrm{OH})_{\mathrm{s}}$ & -0.03 & 4.16 & 1.78 & 0.75 & 0.73 & $\mathrm{R} 3$ \\
$\mathrm{Fe}-\mathrm{Ni}(\mathrm{OH})_{\mathrm{s}}$ & 0.16 & 4.30 & 2.21 & 0.93 & 0.62 & $\mathrm{R} 4$ \\
$\mathrm{Fe}-\mathrm{Cu}(\mathrm{OH})_{\mathrm{s}}$ & 0.02 & 4.33 & 2.18 & 0.82 & 0.59 & $\mathrm{R} 4$ \\
$\mathrm{Co}-\mathrm{Co}(\mathrm{OH})_{\mathrm{s}}$ & 0.12 & 4.50 & 2.65 & 1.31 & 0.42 & $\mathrm{R} 4$ \\
$\mathrm{Co}-\mathrm{Ni}(\mathrm{OH})_{\mathrm{s}}$ & 0.29 & 4.67 & 2.75 & 1.32 & 0.25 & $\mathrm{R} 4$ \\
$\mathrm{Pt}-\mathrm{Co}(\mathrm{OH})_{\mathrm{s}}$ & 0.18 & 4.57 & 2.68 & 1.39 & 0.35 & $\mathrm{R} 4$ \\
Table S1 & & & & & & \multicolumn{2}{|}{$\mathbf{X u}$ et al. }
\end{tabular}

